\title{
Replication analysis of genetic association of the NCAN-CILP2 region with plasma lipid levels and non-alcoholic fatty liver disease in Asian and Pacific ethnic groups
}

Supichaya Boonvisut', Kazuhiro Nakayama', Saho Makishima', Kazuhisa Watanabe', Hiroshi Miyashita², Munkhtulga Lkhagvasuren ${ }^{3}$, Yasuo Kagawa ${ }^{4}$ and Sadahiko Iwamoto ${ }^{{ }^{*}}$

\begin{abstract}
Background: The Neurocan-cartilage intermediate layer protein 2 (NCAN-CILP2) region forms a tight linkage disequilibrium (LD) block and is associated with plasma lipid levels and non-alcoholic fatty liver disease (NAFLD) in individuals of European descent but not in the Malay and Japanese ethnic groups. Recent genome-wide resequence studies identified a missense single-nucleotide polymorphism (SNP) (rs58542926) of the transmembrane 6 superfamily member 2 (TM6SF2) gene in the NCAN-CILP2 region related to hepatic triglyceride content. This study aims to analyze the influences of SNPs in this region on NAFLD and plasma lipid levels in the Asian and Pacific ethnic groups and to reveal the reasons behind positive and negative genetic associations dependent on ethnicity.

Methods: Samples and characteristic data were collected from 3,013 Japanese, 119 Palauan, 947 Mongolian, 212 Thai and 401 Chinese people. Hepatic sonography data was obtained from the Japanese individuals. Genotyping data of five SNPs, rs58542926, rs735273, rs1009136, rs1858999, and rs16996148, were used to verify the effect on serum lipid levels by multiple linear regression, and the association with NAFLD in the Japanese population was examined by logistic regression analysis.
\end{abstract}

Results: rs58542926 showed significant association with the plasma triglyceride (TG) level in Japanese ( $P=0.0009$, effect size $=9.5( \pm 3.25) \mathrm{mg} / \mathrm{dl} / \mathrm{allele})$ and Thai $(P=0.0008$, effect size $=31.6( \pm 11.7) \mathrm{mg} / \mathrm{dl} / \mathrm{allele})$ study subjects. In Mongolian individuals, there was a significant association of rs58542926 with total cholesterol level $(P=0.0003$, $11.7( \pm 3.2) \mathrm{mg} / \mathrm{dl} / \mathrm{allele}$ ) but not with TG level. In multiple comparisons in Chinese individuals, rs58542926 was weakly $(P=0.022)$ associated with TG levels, although the threshold for statistical significance was not reached. In Palauan individuals, there was no significant association with the studied SNPs. rs58542926 also showed significant association with Japanese NAFLD. The minor allele (t) increased NAFLD risk (OR 1.682, 95 \% Cl 1.289-2.196, $p$ value 0.00013).

Conclusion: This study confirmed the genetic association of missense SNP of TM6SF2, rs58542926, with plasma lipid levels in multiple East Asian ethnic groups and with NAFLD in Japanese individuals.

Keywords: TM6SF2, Asian and Oceanian populations, Triglyceride, Cholesterol, Fatty liver disease

\footnotetext{
* Correspondence: siwamoto@jichi.ac.jp

'Division of Human Genetics, Center for Molecular Medicine, Jichi Medical

University, 3311-1 Yakushiji, Shimotsuke, Tochigi 329-0498, Japan

Full list of author information is available at the end of the article
} 


\section{Background}

Dyslipidemia, which refers to abnormally high or low lipid levels in circulating blood, is a major heritable risk factor of cardiovascular and cerebrovascular diseases. Many studies have investigated genetic factors of plasma lipid levels to explore the responsible metabolic pathways. Through genome-wide association studies (GWAS), abundant genetic variants associated with plasma lipid levels have been identified, among which the Neurocan-cartilage intermediate layer protein 2 (NCAN-CILP2) region has high statistical significance and a relatively large effect size per allele on low-density lipoprotein cholesterol (LDL) and triglyceride (TG) levels $[1,2]$. The NCAN-CILP2 region spans $300 \mathrm{~kb}$ on chromosome 19 and forms a tight linkage disequilibrium (LD) block. Eleven genes and one miRNA are encoded in this region. This locus showed consistent and deep association with serum lipid levels in the subsequent studies for individuals of European and Chinese descent [3-5]. However, the tag SNP in this region, rs16996148, was not correlated with plasma lipids in Malaysian [6] and Japanese populations $[7,8]$.

In addition to plasma lipid levels, the NCAN-CILP2 region was identified as a non-alcoholic fatty liver disease (NALFD)-associated locus by GWAS in individuals of European descent [9] but not in Japanese individuals $[10,11]$. The risk allele for NAFLD in this region decreased plasma lipid levels in individuals of European descent [9]. Recent genome-wide resequence studies identified a missense single-nucleotide polymorphism (SNP) (rs58542926) in transmembrane 6 superfamily member 2 (TM6SF2), which is related to hepatic triglyceride content $[12,13]$. Functional assessment of Tm6sf2 in mouse and human hepatocytes revealed that it regulated plasma TG and cholesterol levels in mice and lipid accumulation in hepatocytes [12-14].

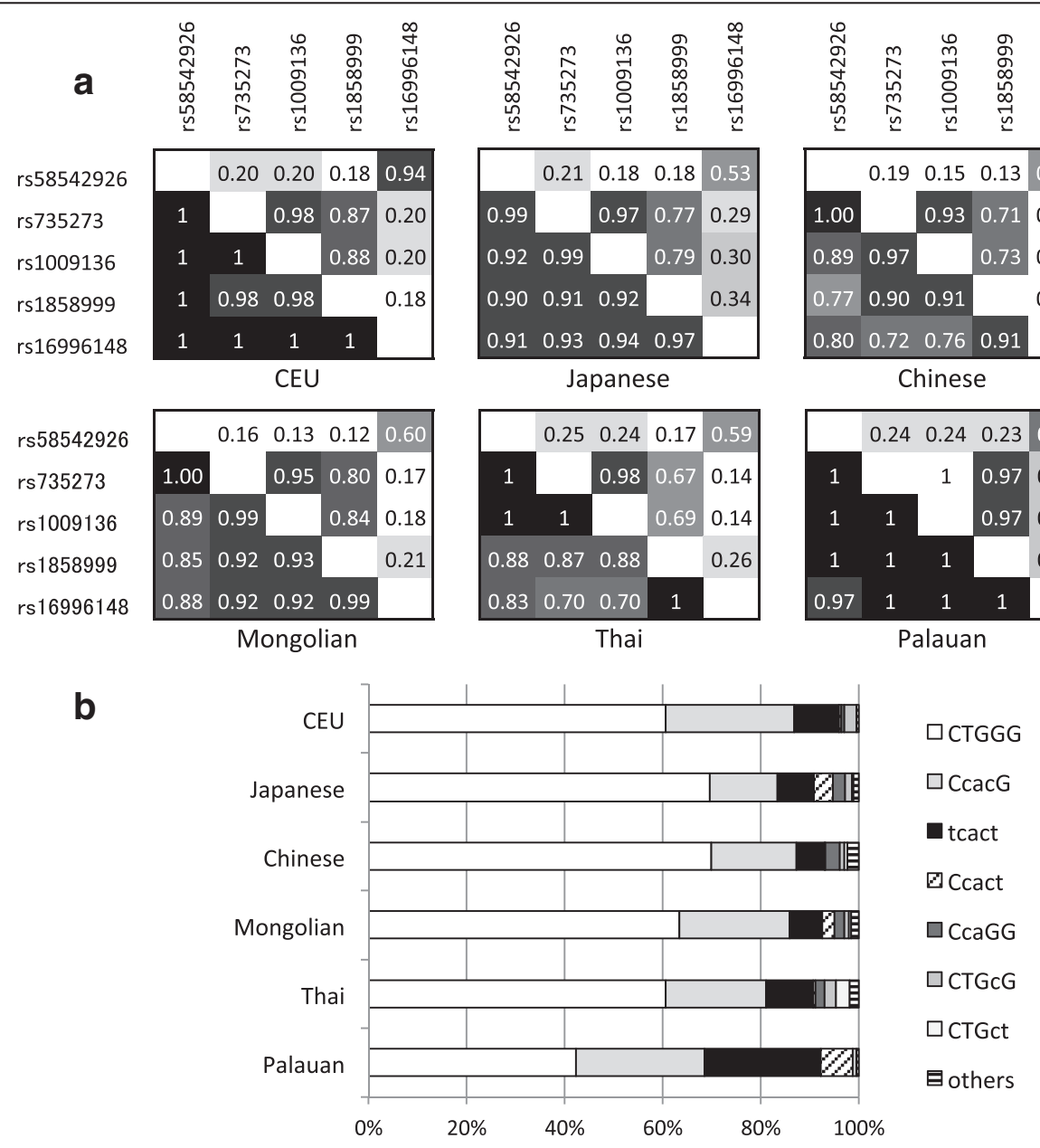

Fig. 1 a Pairwise linkage disequilibrium analysis of the five studied SNPs in the NCAN-CILP2 region in European (CEU), Japanese, Chinese, Mongolian, Thai and Palauan individuals. Numerals in the left-lower diagonal show $\left|\mathrm{D}^{\prime}\right|$ values and in the right-upper diagonal half show $r^{2}$ values. $\mathbf{b}$ Haplotype frequencies in the five studied populations and referential CEU population. The chart on the right shows the estimated haplotypes in the order of rs58542926, rs735273, rs1009136, rs1858999 and rs16996148. Capital letters indicate major alleles, and small letters indicate minor alleles 
rs16996148 and rs58542926 are on opposite sides of the LD block. There was no significant difference in LD structure between European and Japanese populations in the HapMap database (http://hapmap.ncbi.nlm.nih.gov/). The present study aims to analyze the influences of SNPs in this region on NAFLD and plasma lipid levels in Asian and Pacific ethnic groups and to reveal the reasons behind positive and negative genetic correlations dependent on ethnicity.

\section{Results}

Genetic association of NCAN-CILP2 region with East Asian plasma lipid levels

Genotyping success rates were $>97 \%$ for all of the SNPs. There were no deviations from Hardy-Weinberg equilibrium for any of the SNPs in all ethnic groups $(P>0.05)$. Details of all 5 SNPs and minor allele frequencies (MAFs) for each population are described in Table 1. rs58542926 showed significant associations with plasma lipid levels in multiple ethnic groups, which persisted after applying the Bonferroni correction for multiple testing (5 SNPs and 4 phenotypes). In Japanese individuals, rs58542926 was strongly associated with TG level. The minor allele ( $\mathrm{t}$ ) decreased the TG level by 9.5 $( \pm 3.25) \mathrm{mg} / \mathrm{dl} /$ allele. rs58542926, rs735273, rs1009136 and rs1858999 were weakly associated with TG level in Chinese individuals, but the associations did not reach the statistical significance threshold. In Mongolian individuals, a significant association of rs58542926 was observed with total cholesterol and LDL level, but not with TG level. The minor allele decreased total cholesterol by $11.7( \pm 3.2) \mathrm{mg} / \mathrm{dl} /$ allele and LDL cholesterol by 8.3 $( \pm 2.9) \mathrm{mg} / \mathrm{dl} /$ allele. However, the minor allele solely decreased the TG level in Thai individuals by $31.6( \pm 11.7)$ $\mathrm{mg} / \mathrm{dl} /$ allele. In Palauan individuals, there were no significant associations with the studied SNPs. In combined analysis, the association of rs58542926 with total cholesterol and TG levels becomes further significant. These results support the previous data that the studied missense SNP in TM6SF2 is the functional SNP in the NCAN-CILP2 region.

\section{Genetic association of the NCAN-CILP2 region with Japanese NAFLD}

Among the studied SNPs, rs58542926 showed the most significant association with Japanese NAFLD. The minor allele $(\mathrm{t}$ ) increased NAFLD risk (OR 1.682, 95 \% CI 1.2892.196, $p$ value 0.00013 ) (Table 2). The allele that reduced plasma lipid levels was the risk allele for NAFLD.

\section{Haplotype frequency and linkage disequilibrium structures}

To evaluate the LD structure of NCAN-CILP2 in the Asian and Pacific populations, a pairwise linkage disequilibrium map was drawn for each ethnic group, and the maps were compared with $\left|\mathrm{D}^{\prime}\right|$ and $r^{2}$ values of the CEU population downloaded from the HapMap database (Fig. 1a). The middle three SNPs, rs735273, rs1009136, and rs1858999, showed relatively high $r^{2}$ values in all of the ethnic groups. But, the most telomeric side SNP, rs58542926, showed weak LD with the middle LD block, even though the physical distance between rs58542926 and rs735273 was close, $5.8 \mathrm{~kb}$, and they showed a high $\left|D^{\prime}\right|$ value, indicating rare recombinations between them. Conversely, the most centromeric side SNP, rs16996148, showed a moderate $r^{2}$ score with rs58542926, despite the long distance between them, $289 \mathrm{~kb}$. The $r^{2}$ score between rs58542926 and rs16996148 in the CEU population was relatively high. The estimated haplotype frequency showed a higher prevalence of the haplotype encoding the NAFLD risk allele in the Palauan individuals as compared to other ethnic groups (Fig. 1b). Haplotypes carrying minor allele (t) of rs16996148 in the CEU population mostly (94.7\%) encoded as minor allele (t) of rs58542926, but in the Japanese, Mongolian, Thai and Palauan populations, substantial haplotypes (21.5$37.1 \%$ ) encoded major allele (C) of rs58542926 with (t) of rs16996148.

\section{Discussion}

This study replicated the genetic association of a missense SNP in TM6SF2 with plasma lipid levels in multiple ethnic groups, while the other four SNPs did not show significant associations. Lysine at residue 167 in TM6SF2 reduced TG but did not reduce cholesterol in the plasma of Japanese and Thai individuals. In Mongolian individuals, rs58542926 reduced total cholesterol but not TG. Despite the higher minor allele frequency in Palauan individuals, rs58542926 was not associated with lipid levels. A small population size was considered as the main cause of the lack of association, but other ethnic-specific factors could have affected the results (e.g., relatively higher prevalence of type 2 diabetes (20.2 \%) and obesity $(87.8 \%$, body mass index $(\mathrm{BMI})>25))$ in the Palauan population [15]. Population-dependent preference to associated lipid fraction was also observed in previous studies. Norwegians showed association only with total cholesterol, [12] but European Americans showed over 400-fold smaller $\mathrm{p}$ value with TG than LDL cholesterol [13]. The Hispanic group did not show any significant association with lipid levels [13]. These results suggest that the regulation of cholesterol and TG in plasma by TM6SF2 is affected by ethnicity. The variable effect of rs58542926 on plasma lipid profiles may be feasibly explained by differences in dietary fat or neutraceutical intake [16] according to ethnic tradition.

This study also replicated the genetic association of TM6SF2 with NAFLD in Japanese. The lysine allele of rs58542926 increased the risk diagnosed of a bright liver 
Table 1 Association study of SNPs in NCAN-CILP2 region with serum lipid levels in East Asian ethnic groups

\begin{tabular}{|c|c|c|c|c|c|c|c|c|c|c|c|c|c|}
\hline \multicolumn{2}{|l|}{$\begin{array}{l}\text { dbSNP ID position } \\
\text { Nearest gene Allele }\end{array}$} & \multirow{2}{*}{\multicolumn{2}{|c|}{$\begin{array}{l}\begin{array}{l}\text { Japanese } \\
(\mathrm{n}=3014)\end{array} \\
0.080(0.858)\end{array}$}} & \multirow{2}{*}{\multicolumn{2}{|c|}{$\begin{array}{l}\text { Chinese } \\
(n=401)\end{array}$}} & \multirow{2}{*}{\multicolumn{2}{|c|}{$\begin{array}{l}\text { Mongol }(n=947) \\
0.078(0.707)\end{array}$}} & \multirow{2}{*}{\multicolumn{2}{|c|}{$\begin{array}{l}\text { Thai }(n=212) \\
0.114(0.227)\end{array}$}} & \multirow{2}{*}{\multicolumn{2}{|c|}{$\begin{array}{l}\text { Palau }(n=119) \\
0.237(0.663)\end{array}$}} & \multicolumn{2}{|c|}{$\begin{array}{l}\text { Combined } \\
(\mathrm{n}=4693)\end{array}$} \\
\hline \multirow{2}{*}{$\begin{array}{l}\text { rs58542926 chr19: } \\
19268740\end{array}$} & \multirow[t]{2}{*}{ MAF (P-HWE) } & & & & & & & & & & & & \\
\hline & & $\beta$ & $P$ value & $\beta$ & $P$ value & $\beta$ & $P$ value & $e \beta$ & $P$ value & $\beta$ & $P$ value & $\beta$ & $P$ value \\
\hline TM6SF2 & total cholesterol & -0.035 & 0.048 & -0.089 & 0.0835 & -0.116 & $0.0003^{*}$ & * -0.095 & 0.157 & 0.012 & 0.907 & -0.051 & $0.0005^{*}$ \\
\hline E167K & LDL & -0.027 & 0.130 & -0.041 & 0.4276 & -0.091 & 0.005 & -0.077 & 0.258 & 0.077 & 0.454 & -0.030 & 0.039 \\
\hline \multirow[t]{2}{*}{$\mathrm{C} / \mathrm{t}$} & $\mathrm{HDL}$ & 0.005 & 0.747 & 0.0265 & 0.5837 & -0.018 & 0.551 & 0.107 & 0.109 & -0.128 & 0.195 & -0.007 & 0.600 \\
\hline & $\log T G$ & -0.054 & $0.0009^{*}$ & -0.109 & 0.0224 & -0.030 & 0.334 & -0.222 & $0.0008^{*}$ & -0.023 & 0.813 & -0.056 & $3.15 \times 10^{-5^{*}}$ \\
\hline rs735273 & MAf (P-HWE) & \multicolumn{2}{|c|}{$0.291(0.719)$} & \multicolumn{2}{|c|}{$0.277(0.613)$} & \multicolumn{2}{|c|}{$0.351(0.788)$} & \multicolumn{2}{|c|}{$0.342(0.151)$} & \multicolumn{2}{|c|}{$0.572(0.966)$} & & \\
\hline chr19:19274602 & & $\beta$ & $P$ value & $\beta$ & $P$ value & $\beta$ & $P$ value & $e \beta$ & $P$ value & $\beta$ & $P$ value & $\beta$ & $P$ value \\
\hline TM6SF2 & total cholesterol & 0.008 & 0.674 & -0.091 & 0.071 & -0.034 & 0.293 & -0.018 & 0.787 & -0.049 & 0.639 & -0.030 & 0.036 \\
\hline 5' flanking & LDL & -0.005 & 0.793 & -0.019 & 0.700 & 0.001 & 0.986 & 0.024 & 0.724 & -0.008 & 0.936 & -0.019 & 0.181 \\
\hline \multirow[t]{2}{*}{$T / C$} & $\mathrm{HDL}$ & 0.037 & 0.019 & -0.017 & 0.728 & -0.038 & 0.211 & 0.024 & 0.721 & -0.048 & 0.635 & -0.005 & 0.716 \\
\hline & $\log T G$ & -0.025 & 0.128 & -0.098 & 0.038 & -0.019 & 0.544 & -0.147 & 0.028 & -0.052 & 0.599 & -0.031 & 0.019 \\
\hline rs1009136 & MAF (P-HWE) & \multicolumn{2}{|c|}{$0.286(0.757)$} & \multicolumn{2}{|c|}{$0.275(0.706)$} & \multicolumn{2}{|c|}{$0.341(0.911)$} & \multicolumn{2}{|c|}{$0.347(0.128)$} & \multicolumn{2}{|c|}{$0.572(0.966)$} & & \\
\hline chr19:19329619 & & $\beta$ & $P$ value & $\beta$ & $P$ value & $\beta$ & $P$ value & $e \beta$ & $P$ value & $\beta$ & $P$ value & $\beta$ & $P$ value \\
\hline MAU2 & total cholesterol & 0.008 & 0.663 & -0.088 & 0.084 & -0.042 & 0.186 & -0.019 & 0.783 & -0.049 & 0.693 & -0.030 & 0.041 \\
\hline intronic & LDL & -0.005 & 0.793 & -0.005 & 0.915 & -0.001 & 0.977 & 0.021 & 0.755 & -0.008 & 0.936 & -0.016 & 0.275 \\
\hline \multirow[t]{2}{*}{ G/a } & $\mathrm{HDL}$ & 0.038 & 0.018 & -0.009 & 0.855 & -0.041 & 0.178 & 0.036 & 0.590 & -0.048 & 0.635 & -0.005 & 0.719 \\
\hline & $\log$ TG & -0.026 & 0.113 & -0.094 & 0.047 & -0.031 & 0.313 & -0.148 & 0.027 & -0.058 & 0.599 & -0.035 & 0.010 \\
\hline rs1858999 & MAF (P-HWE) & \multicolumn{2}{|c|}{$0.276(0.844)$} & \multicolumn{2}{|c|}{$0.253(0.349)$} & \multicolumn{2}{|c|}{$0.335(0.422)$} & \multicolumn{2}{|c|}{$0.370(0.168)$} & \multicolumn{2}{|c|}{$0.5812(0.994)$} & & \\
\hline chr19:19386860 & & $\beta$ & $P$-value & $\beta$ & $P$-value & $\beta$ & $P$-value & $\beta$ & $P$-value & $\beta$ & $P$-value & $\beta$ & $P$-value \\
\hline GATAD2A & total cholesterol & -0.003 & 0.853 & -0.098 & 0.054 & -0.047 & 0.139 & -0.023 & 0.729 & -0.053 & 0.610 & -0.037 & 0.012 \\
\hline intronic & LDL & -0.019 & 0.289 & -0.003 & 0.946 & 0.000 & 0.997 & -0.008 & 0.909 & -0.010 & 0.927 & -0.022 & 0.121 \\
\hline $\mathrm{G} / \mathrm{C}$ & $\mathrm{HDL}$ & 0.046 & 0.004 & 0.008 & 0.866 & -0.031 & 0.308 & 0.035 & 0.594 & -0.067 & 0.501 & 0.001 & 0.997 \\
\hline & $\log T G$ & -0.044 & 0.007 & -0.110 & 0.021 & -0.027 & 0.380 & -0.112 & 0.096 & -0.060 & 0.541 & -0.042 & $0.002^{*}$ \\
\hline rs16996148 & MAF (P-HWE) & $0.119(0$ & .470) & $0.069(0$ & $0.611)$ & $0.099(0$. & & $0.132(0$ & $0.631)$ & 0.308 & .854) & & \\
\hline chr19:19547663 & & $\beta$ & $P$ value & $\beta$ & $P$ value & $\beta$ & $P$ value & $e \beta$ & $P$ value & $\beta$ & $P$ value & $\beta$ & $P$ value \\
\hline CILP2 & total cholesterol & -0.034 & 0.057 & -0.049 & 0.336 & -0.092 & 0.004 & -0.106 & 0.116 & 0.024 & 0.820 & -0.032 & 0.026 \\
\hline 3' flanking & LDL & -0.032 & 0.069 & 0.005 & 0.923 & -0.073 & 0.024 & -0.094 & 0.166 & 0.123 & 0.238 & -0.016 & 0.276 \\
\hline $\mathrm{G} / \mathrm{t}$ & $\mathrm{HDL}$ & 0.009 & 0.554 & 0.030 & 0.538 & -0.049 & 0.106 & 0.091 & 0.171 & -0.080 & 0.421 & 0.001 & 0.948 \\
\hline & $\log$ TG & -0.038 & 0.020 & -0.065 & 0.175 & -0.007 & 0.817 & -0.181 & 0.007 & -0.058 & 0.552 & -0.037 & 0.006 \\
\hline
\end{tabular}

Beta coefficient ( $\beta$ ) and $P$ values of copy numbers of minor alleles for each lipid parameter in multiple linear regression models of the indicated ethnic groups are shown. Minor alleles of each SNP are indicated by lowercase letters. Statistical significance is shown by an asterisk (*) on the threshold of $<0.0025$. MAF (P HWE): minor allele frequency ( $P$ value of Hardy-Weinberg equilibrium.)

Table 2 Association study of SNPs in NCAN-CILP2 region with NAFLD in Japanese individuals

\begin{tabular}{llll}
\hline dbSNP ID (nearest gene) & OR & $(95 \% \mathrm{Cl})$ & $p$ value \\
\hline rs58542926 (TM6SF2) & 1.682 & $(1.289-2.196)$ & 0.001 \\
rs735273 (TM6SF2) & 1.290 & $(1.093-1.522)$ & 0.003 \\
rs1009136 (MAU2) & 1.251 & $(1.060-1.478)$ & 0.008 \\
rs1858999 (GATAD2A) & 1.199 & $(1.015-1.417)$ & 0.033 \\
rs16996148 (CILP2) & 1.186 & $(0.943-1.493)$ & 0.145 \\
\hline
\end{tabular}

Odd ratio $(\mathrm{OR})$ and $\mathrm{P}$ value of each minor allele in the logistic regression model are shown in ultrasonography. Although the exact biochemical function of TM6SF2 protein has not yet been revealed, knocking down of Tm6sf2 expression in mouse liver reduced cholesterol and TG levels in mouse plasma and increased hepatic lipid droplet content [12, 13]. In human hepatocyte cell lines, disturbed expression of TM6SF2 reduced VLDL excretion and increased cytoplasmic lipid droplets [14]. Furthermore, amino acid substitution at residue 167 from glutamine to lysine diminished the protein level in the endoplasmic reticulum membrane of the transfected cells, probably via misfolded protein degradation [13]. Therefore, it was suggested that TM6SF2 protein is involved in lipid 
transportation through the ER membrane. Our genetic results support the theory that the SNP induced a change of the physiological function of the TM6SF2 gene.

rs16996148 was initially identified as an SNP associated with plasma cholesterol and TG in a population of European descent [2]. However, this finding was not replicated in Japanese individuals [7]. The LD score between rs16996148 and rs58542926 in the CEU population was more rigid than in East Asian populations, due to the low frequencies of Ccact and CTGct haplotypes. This distinct LD structure and haplotype frequencies in East Asian populations may have resulted in the lack of association. The haplotype frequency in our studied ethnic groups suggested that the functional SNP in TM6SF2, rs58542926, emerged in the haplotype encoding minor alleles at the remaining four SNPs. It was intriguing that the minor allele haplotype was highly prevalent in Palauan individuals as compared to other ethnic groups. Although rs58542926 is a risk allele for NAFLD, it has been reported to be beneficial for CAD [12, 17]. The NCAN-CILP2 region was also suspected to be a type 2 diabetes-associated locus [18], although the pathogenic locus has not been identified. One explanation for these observations may be that the NAFLD risk allele was dramatically increased in the ancestors of Palauan individuals by natural selection, which would be related to energy metabolism. The NAFLD risk allele may be associated with a phenotype that stores excessive energy in the liver and might be advantageous under conditions of highly restricted food availability. Further population genetic studies are required to determine if the higher prevalence of the Lys167 allele in the Palauan population is a consequence of natural selection or simple genetic drift.

\section{Conclusion}

In conclusion, our study replicated the genetic association of missense SNP of TM6SF2, rs58542926, with plasma lipid levels in East Asian multiple ethnic groups. While associated lipid, cholesterol, or TG levels were dependent on ethnicity, the effect sizes were relatively large. The SNP was also associated with Japanese NAFLD. These results support previous genetic studies and can be used to estimate the physiological function of TM6SF2, which could be a personal biomarker for medical management of dyslipidemia and NAFLD.

\section{Methods}

\section{Study populations}

The present study design was approved by the ethical committee of Jichi Medical University and Kagawa Nutrition University. All participants provided written informed consent. Samples were collected from 3,013 Japanese, 119 Palauan, 947 Mongolian, 212 Thai and
401 Chinese people. Population characteristics have been previously described $[15,19]$. Participant age, sex, alcohol consumption (obtained by self-questionnaire), body mass index (BMI), and levels of plasma triglyceride (TG), total cholesterol, low-density lipoprotein cholesterol (LDL), and high-density lipoprotein cholesterol (HDL-c) (mg/dl) were collected. Hepatic sonography data were simultaneously obtained in Japanese samples for fatty liver evaluation, which was examined by an experienced clinical technologist and reviewed by clinicians.

\section{Genotyping and association analysis}

The following five SNPs of the NCAN-CILP2 LD block were chosen considering the LD structure of the Japanese population and the results of recent studies [9-13]: rs58542926 (most adjacent gene; TM6SF2), rs735273 (TM6SF2), rs1009136 (MAU2), rs1858999 (GATAD2A), and rs16996148 (CILP2). All five SNPs were genotyped by the TaqMan Genotyping Assay Systems (Applied Biosystems, Foster City, CA, USA). Multiple linear regression analysis was employed to calculate the effect of each minor allele on four serum lipid concentration levels: TG, total cholesterol, LDL, and HDL. The analysis was calculated separately in each ethnic group. Age, sex, and BMI were included for adjustment as covariant factors. Triglyceride levels were transformed to logarithm to reduce the overestimated effect from skewed distribution, but untransformed values of triglycerides were also assessed for the purpose of showing an effect size of SNPs. To study the association of these five SNPs and NAFLD in the Japanese population, participants who had excessive alcohol consumption ( $>20 \mathrm{~g} /$ day) history were excluded. Then, genotyping results of 2,281 of the 3,013 Japanese DNA panels were studied. Logistic regression analysis was applied with adjustments for age, sex, BMI, and type 2 diabetes mellitus (DM2). All statistical analyses were performed with SPSS software. Haplotype frequencies for each population were calculated, and LD blocks were drawn by Haploview software [20].

\section{Abbreviations \\ BMI: body mass index; DM2: type 2 diabetes mellitus; GWAS: genome-wide association studies; HDL-c: high-density lipoprotein cholesterol; LD: linkage disequilibrium; LDL: low-density lipoprotein cholesterol; MAF: minor allele frequency; NAFLD: non-alcoholic fatty liver disease; NCAN-CILP2: Neurocan- cartilage intermediate layer protein 2; SNP: single-nucleotide polymorphism; TG: triglyceride; TM6SF2: transmembrane 6 superfamily member 2.}

\section{Competing interests}

The authors declare that they have no competing interests.

\section{Authors' contributions}

SB: designed and performed experiments, analyzed data, wrote and revised manuscript; KN, YK: collected and analyzed data, wrote and revised

manuscript; SM, KW, HM, ML: collected data and prepared experiments; SI: designed study, analyzed data, and critically revised manuscript. All authors read and approved the final manuscript. 


\section{Acknowledgements}

This study was supported by grant-in-aid (Nos. 25461377 and 26291096) from the Japanese Ministry of Education, Culture, Sports, Science and Technology (MEXT); a MEXT-supported program for the strategic research foundation at private universities (2013-2017); and a grant from the Takeda Science Foundation (No. 3-2313-004), Osaka, Japan. We are grateful to Kayo Nagashima and Yukiko Ohashi for their excellent technical assistance.

\section{Author details}

'Division of Human Genetics, Center for Molecular Medicine, Jichi Medical University, 3311-1 Yakushiji, Shimotsuke, Tochigi 329-0498, Japan. ${ }^{2}$ Jichi Medical University Health Care Center, Shimotsuke, Tochigi 329-0498, Japan ${ }^{3}$ School of Medicine, Mongolian National University, Bayagol district, 11th khoroo, Ulaanbaatar-city, Mongolia. ${ }^{4}$ High Technology Research Center, Kagawa Nutrition University, Sakado, Saitama 350-0288, Japan.

Received: 21 November 2015 Accepted: 8 January 2016 Published online: 13 January 2016

\section{References}

1. Willer CJ, Sanna S, Jackson AU, Scuteri A, Bonnycastle LL, Clarke R, et al. Newly identified loci that influence lipid concentrations and risk of coronary artery disease. Nat Genet. 2008;40:161-9.

2. Kathiresan S, Melander O, Guiducci C, Surti A, Burtt NP, Rieder MJ, et al. Six new loci associated with blood low-density lipoprotein cholesterol, high-density lipoprotein cholesterol or triglycerides in humans. Nat Genet 2008:40:189-97.

3. Waterworth DM, Ricketts SL, Song K, Chen L, Zhao JH, Ripatti S, et al. Genetic variants influencing circulating lipid levels and risk of coronary artery disease. Arterioscler Thromb Vasc Biol. 2010;30(11):2264-76.

4. Keebler ME, Deo RC, Surti A, Konieczkowski D, Guiducci C, Burtt N, et al. Fine-Mapping in African Americans of Eight Recently Discovered Genetic Loci for Plasma Lipids: The Jackson Heart Study. Circ Cardiovasc Genet. 2010;3(4):358-64

5. Yan $T$, Yin RX, Li Q, Huang $P$, Zeng XN, Huang KK, et al. Sex-specific association of rs16996148 SNP in the NCAN/CILP2/PBX4 and serum lipid levels in the Mulao and Han populations. Lipids Health Dis. 2011:10:248.

6. Tai ES, Sim XL, Ong TH, Wong TY, Saw SM, Aung T, et al. Polymorphisms at newly identified lipid-associated loci are associated with blood lipids and cardiovascular disease in an Asian Malay population. J Lipid Res. 2009;50(3): 514-20.

7. Kamatani Y, Matsuda K, Okada Y, Kubo M, Hosono N, Daigo Y, et al. Genome-wide association study of hematological and biochemical traits in a Japanese population. Nat Genet. 2010:42:210-5.

8. Nakayama K, Bayasgalan T, Yamanaka K, Kumada M, Gotoh T, Utsumi N, et al. Large scale replication analysis of loci associated with lipid concentrations in a Japanese population. J Med Genet. 2009:46:370-4.

9. Speliotes EK, Yerges-Armstrong LM, Wu J, Hernaez R, Kim LJ, Palmer CD, et al. Genome-wide association analysis identifies variants associated with nonalcoholic Fatty liver disease that have distinct effects on metabolic traits. PLoS Genet. 2011;7:e1001324.

10. Kawaguchi T, Sumida Y, Umemura A, Matsuo K, Takahashi M, Takamura T, et al. Genetic Polymorphisms of the Human PNPLA3 Gene Are Strongly Associated with Severity of Non-Alcoholic Fatty Liver Disease in Japanese. PLoS One. 2012;7(6):e38322.

11. Kitamoto T, Kitamoto A, Yoneda M, Hyogo H, Ochi H, Nakamura T, et al. Genome-wide scan revealed that polymorphisms in the PNPLA3, SAMM50, and PARVB genes are associated with development and progression of nonalcoholic fatty liver disease in Japan. Hum Genet. 2013;132:783-92.

12. Holmen OL, Zhang H, Fan $Y$, Hovelson DH, Schmidt EM, Zhou W, et al. Systematic evaluation of coding variation identifies a candidate causal variant in TM6SF2 influencing total cholesterol and myocardial infarction risk. Nat Genet. 2014;46(4):345-51.

13. Kozlitina J, Smagris E, Stender S, Nordestgaard BG, Zhou HH, TybjærgHansen A, et al. Exome-wide association study identifies a TM6SF2 variant that confers susceptibility to nonalcoholic fatty liver disease. Nat Genet. 2014;46:352-6

14. Mahdessian H, Taxiarchis A, Popov S, Silveira A, Franco-Cereceda A Hamsten A, et al. TM6SF2 is a regulator of liver fat metabolism influencing triglyceride secretion and hepatic lipid droplet content. Proc Natl Acad Sc U S A. 2014;111:8913-8.
15. Nakayama K, Yanagisawa Y, Ogawa A, Ishizuka Y, Munkhtulga L, Charupoonphol $\mathrm{P}$, et al. High prevalence of an anti-hypertriglyceridemic variant of the MLXIPL gene in Central Asia. J Hum Genet. 2011;56(12):828-33.

16. Scicchitano P, Cameli M, Maiello M, Modesti PA, Muiesan ML, Novo S, et al. Nutraceuticals and dyslipidaemia: Beyond the common therapeutics. J Funct Food. 2014;6:11-32.

17. Zhou L, Ding H, Zhang X, He M, Huang S, Xu Y, et al. Genetic Variants at Newly Identified Lipid Loci Are Associated with Coronary Heart Disease in a Chinese Han Population. PLoS One. 2011;6(11):e27481.

18. Saxena R, Elbers CC, Guo Y, Peter I, Gaunt TR, Mega JL, et al. Large-scale gene-centric meta-analysis across 39 studies identifies type 2 diabetes loci. Am J Hum Genet. 2012;90(3):410-25.

19. Nakayama K, Ogawa A, Miyashita H, Tabara Y, Igase M, Kohara K, et al. Positive natural selection of TRIB2, a novel gene that influences visceral fat accumulation, in East Asia. Hum Genet. 2013:132(2):201-17.

20. Barrett JC, Fry B, Maller J, Daly MJ. Haploview: analysis and visualization of LD and haplotype maps. Bioinformatics. 2005:21(2):263-5.

\section{Submit your next manuscript to BioMed Central and we will help you at every step:}

- We accept pre-submission inquiries

- Our selector tool helps you to find the most relevant journal

- We provide round the clock customer support

- Convenient online submission

- Thorough peer review

- Inclusion in PubMed and all major indexing services

- Maximum visibility for your research

Submit your manuscript at www.biomedcentral.com/submit 